\title{
PROOF OF A CONJECTURE ABOUT MEASURABLE SETS ON THE REAL LINE
}

\section{J. J. SCHÄFFER}

In $[1$, p. 243] the author formulated a conjecture concerning Lebesgue-measurable sets on the real line; this conjecture was connected with an analysis of the structure of a class of translationinvariant function spaces. The purpose of this note is to give a proof of that conjecture. The proof is based on an idea of E. Calabi. The research was carried out while the author held a fellowship granted by the John Simon Guggenheim Memorial Foundation.

Let $R$ denote the real line and let $\mu$ be Lebesgue measure on $R$. The sign $\backslash$ is used to denote set-theoretical differences. If $F, F^{\prime} \subset R$ are $\mu$-measurable, we say that $F$ is essentially contained in $F^{\prime}$ if $\mu\left(F \backslash F^{\prime}\right)=0$.

THEOREM. If $E \subset R$ is $\mu$-measurable and $\mu(E)>0$, there exists a closed, bounded set $E^{\prime} \subset R$ with $\mu\left(E^{\prime}\right)>0$, such that $E$ is not essentially contained in any finite union of translates of $E^{\prime}$.

Proof. For every measurable set $F \subset R$ with $\mu(F)>0$ and for every real $s>0$ we define

$$
\omega(s ; F)=s^{-1} \cdot \inf _{t \in R} \mu(\{(t+[0, s]) \backslash F\}) .
$$

Obviously, $0 \leqq \omega(s ; F) \leqq 1$. Since $\mu(F)>0$, there exists a point $t_{0} \in F$ of density 1 with respect to $F$, and therefore

$$
\lim _{s \rightarrow 0} s^{-1} \cdot \mu\left(\left\{\left(t_{0}+[0, s]\right) \backslash F\right\}\right)=0 ;
$$

a fortiori we have

$$
\lim _{s \rightarrow 0} \omega(s ; F)=0 .
$$

Let $E$ be the given set. On account of (1) we may construct a sequence of positive integers $\{m(n)\}$ such that the following two conditions are satisfied for all $n=1,2, \cdots$ :

$$
\begin{aligned}
m(n+1) & >m(n)+n+1, \\
\omega\left(2^{-m(n)+1} ; E\right) & <2^{-8 n^{2}} .
\end{aligned}
$$

We define the closed set

$$
A=R \backslash \bigcup_{n=1}^{\infty} \bigcup_{r-\infty}^{\infty}\left(2^{-m(n)} r+\left(0,2^{-m(n)-n-1}\right)\right) .
$$

Received by the editors June 24, 1960. 
The open intervals $2^{-m(n)} r+\left(0,2^{-m(n)-n-1}\right), r=0, \pm 1, \pm 2, \cdots$, shall be called the holes of order $n$ of $A$; the images of these holes under the translation $t \rightarrow t^{\prime}+t$ are the holes of order $n$ of $t^{\prime}+A$. We now set

$$
E^{\prime}=[0,1] \cap A,
$$

so that $E^{\prime}$ is closed and bounded, and

$$
\mu\left(E^{\prime}\right) \geqq 1-\sum_{n=1}^{\infty} \sum_{r=0}^{2^{m(n)}-1} 2^{-m(n)-n-1}=1-\sum_{n=1}^{\infty} 2^{-n-1}=1 / 2>0 .
$$

We claim that $E$ is not essentially contained in any finite union of translates of $A$, let alone of $E^{\prime}$. Assume, by contradiction, that $E$ is essentially contained in $B=\bigcup_{j=1}^{k}\left(t_{j}+A\right)$ for some positive integer $k$ and some $t_{1}, \cdots, t_{k} \in R$. From the definition of $\omega(s ; F)$ and from (3) it follows in particular that

$$
\omega\left(2^{-m(k)+1} ; B\right) \leqq \omega\left(2^{-m(k)+1} ; E\right)<2^{-8 k^{2}} .
$$

Let now $I$ be any closed interval of length $2^{-m(k)+1}$; it contains at least one complete hole of order $k$ of $t_{1}+A$; this hole, being of length $2^{-m(k)-k-1}>2^{-m(k+1)}$ (by condition (2)), contains at least $\left(2^{-m(k)-k-1} / 2^{-m(k+1)}\right)-1 \geqq 2^{m(k+1)-m(k)-k-2}$ complete holes of order $k+1$ of $t_{2}+A$; each of these holes, being of length $2^{-m(k+1)-k-2}>2^{-m(k+2)}$ (by condition (2)), contains at least $\left(2^{-m(k+1)-k-2} / 2^{-m(k+2)}\right)-1$ $\geqq 2^{m(k+2)-m(k+1)-k-8}$ complete holes of order $k+2$ of $t_{3}+A$. Continuing in this way, we find certain holes of order $2 k-1$ of $t_{k}+A$ contained in holes of order $2 k-2$ of $t_{k-1}+A, \cdots$, contained in a hole of order $k$ of $t_{1}+A$; the union $H$ of these holes of order $2 k-1$ (each of length $\left.2^{-m(2 k-1)-2 k}\right)$ is therefore in $\Lambda B$. Now

$$
\begin{aligned}
\mu(H) & \geqq 2^{-m(2 k-1)-2 k} \prod_{j=1}^{k-1} 2^{m(k+j)-m(k+j-1)-k-j-1}=2^{-m(k)-\left(3 k^{2}+3 k-2\right) / 2} \\
& \geqq 2^{-m(k)-8 k^{2}+1} .
\end{aligned}
$$

Since $I$ was an arbitrary interval of length $2^{-m(k)+1}$, we have

$$
\omega\left(2^{-m(k)+1} ; B\right) \geqq \mu(H) / \mu(I) \geqq 2^{-8 k^{2}},
$$

and this is in contradiction to (4).

\section{REFERENCE}

1. J. J. Schäffer, Function spaces with translations, Math. Ann. vol. 137 (1959) pp. 209-262.

UNIVERSITY OF URUGUAY AND University of Chicago 